\title{
LOS JÓVENES UNIVERSITARIOS Y LA OPINIÓN PÚBLICA EN ITALIA DURANTE EL '681
}

DOI: http://dx.doi.org/10.1590/2236-3459/76897

\author{
Antonella Cagnolati \\ Universidad de Foggia, Foggia/Foggia, Italia
}

$\cos 8$

\begin{abstract}
Resumen
Uno de los ejemplos más representativos de los nuevos movimientos sociales que surgieron durante los largos años 60 fueron las movilizaciones estudiantiles, principalmente universitarios, que se desarrollaron en Europa y America, con el proposito de dinamizar, democratizar y hacer más justa, libre y solidaria la sociedad occidental. Especialmente intensa fue la oleada de 1968, que logró dimensiones internacionales y un gran impacto mediático y social, hasta el punto de convertirse en un icono, entre el mito y la realidad, en los imaginarios simbólicos y en la memoria colectivas. En este artículo se profundizan las representaciones de la Universidad y la juventud en los imaginaros colectivos italianos configurados por la prensa diaria, prestando especial atención a la organización, los reclamos, las motivaciones y las aspiraciones de las movilizaciones de los estudiantes universitarios, que fueron los protagonistas de la revolución del ' 68 y los artífices, en última instancia, de los cambios operados en las universidades. Las fuentes manejadas en este estudio están constituidas por los editoriales, artículos de opinión y entrevistas sobre el tema objeto de estudio publicados en el principal diario italiano de difusión nacional vigentes durante el '68: il Corriere della Sera.

Palabras clave: prensa diaria, 1968, opinión pública, universidades, Italia.
\end{abstract}

\section{OS JOVENS ESTUDANTES UNIVERSITÁRIOS E A OPINIÃO PÚBLICA NA ITÁLIA DURANTE O ANO '68}

\begin{abstract}
Resumo
Um dos exemplos mais representativos dos novos movimentos sociais que surgiram durante os longos anos 60 foram as mobilizações estudantis, principalmente estudantes universitários, que foram desenvolvidas na Europa e América, com o objetivo de dinamizar, democratizar e tornar mais justas, livres a sociedade ocidental. Especialmente intensa foi a onda de 1968, que alcançou dimensões internacionais e um grande impacto social e social, a ponto de se tornar um ícone, entre mito e realidade, em imaginários simbólicos coletivos e memória. Neste artigo, aprofundam-se as representações da universidade e da juventude nos imaginários coletivos italianos configurados pela imprensa diária, dando especial atenção à organização, às demandas, às motivações e às aspirações das mobilizações dos estudantes universitários, que eram as protagonistas da revolução dos '68 e os artifícios, na última instância, das mudanças operadas nas universidades. As fontes tratadas neste estudo são constituídas por editoriais, artigos de opinião e entrevistas sobre o tema de estudo publicado no principal jornal italiano de difusão nacional vigente em '68: il Corriere
\end{abstract}

\footnotetext{
${ }^{1}$ Esta investigación forma parte de un proyecto más amplio, que tiene como objetivo analizar los procesos de construcción de imaginarios pedagógicos colectivos a través de la prensa diaria en tiempos de cambio político y social en la Europa Mediterránea e Iberoamérica, desarrollado por el Grupo de Investigación de la Universidad de Valladolid (España) Ágora de Educación (http://www.agoradeeducacion.com).
} 
della Sera.

Palavras-chave: jornais diários, 1968, opinião pública, universidades, Itália.

\title{
THE YOUNG UNIVERSITY STUDENTS AND THE PUBLIC OPINION IN ITALY DURING THE '68
}

\begin{abstract}
One of the most representative examples of the new social movements that emerged during the long 60's were student mobilizations, mainly university students, that were developed in Europe and America, with the purpose of dynamizing, democratizing and making fair, free the Western society. Especially intense was the wave of 1968, which achieved international dimensions and a great media and social impact, to the point of becoming an icon, between myth and reality, in collective symbolic imaginaries and memory. In this article the representations of the University and the youth in the Italian collective imaginaries configured by the daily press are deepened, paying special attention to the organization, the demands, the motivations and the aspirations of the mobilizations of the university students, who were the protagonists of the revolution of the ' 68 and the artifices, at last, of the changes operated in the universities. The sources handled in this study are constituted by the editorials, opinion articles and interviews on the subject published in the main Italian newspaper of national diffusion during '68: il Corriere della Sera.
\end{abstract}

Keywords: daily newspapers, 1968, public opinion, universities, Italy.

\section{LES JEUNES ÉTUDIANTS UNIVERSITAIRES ET L'OPINION PUBLIQUE EN ITALIE AU COURS DE '68}

\section{Résumé}

L'un des exemples les plus représentatifs des nouveaux mouvements sociaux qui ont émergé au cours des années 60 ont été des mobilisations d'étudiants, principalement des étudiants universitaires, développés en Europe et en Amérique, dans le but de dynamiser, de démocratiser et de rendre juste et libre la société occidental. La vague de 1968 a été particulièrement intense, qui a atteint des dimensions internationales et un grand impact social et médiatique, au point de devenir une icône, entre le mythe et la réalité, dans les imaginaires symboliques collectifs et la mémoire. Dans cet article, les représentations de l'université et de la jeunesse dans les imaginaires collectifs italiens configurés par la presse quotidienne sont approfondies, en accordant une attention particulière à l'organisation, aux exigences, aux motivations et aux aspirations des mobilisations des étudiants universitaires protagonistes de la révolution des '68 et les artifices, en fin de compte, des changements opérés dans les universités. Les sources traitées dans cette étude sont constituées par des éditoriaux, des articles d'opinion et des interviews publiés dans le principal journal italien de diffusion nationale en vigueur au '68: il Corriere della Sera.

Mots-clés: journaux quotidiens, 1968, opinion publique, les universités, Italie. 


\section{Introducción}

Dodemos decir con seguridad que el '68 es uno de los ejemplos más llamativos de la rebelión planetaria de los estudiantes en contra de la sociedad de la opulencia, un evento que es el punto terminal de una variada y multifacética movilización social de la generación más joven, que se prolongó durante toda la década de los 60, intercalado entre las dos fechas emblemáticas de la rebelión de los estudiantes estadounidenses de la Universidad de Berkeley en California y el Mayo francés. (KURLANSKY, 2004).

En Italia el '68 se manifiesta como resultado inesperado y traumático de una grieta profunda entre los jóvenes y la sociedad como consecuencia de una modernización acelerada y sin gobierno, que había formado la mentalidad generalizada de una nueva generación que creció en la sociedad de consumo y de la comunicación global de masas. (ALDMAN; CHAPMAN; MARWICK, 2000). La incompatibilidad entre la visión del mundo dominada por los valores negativos del capitalismo y el imaginario social de los jóvenes alimentados en mitos anti-autoritarios, igualitarios y pacifistas deflagró en las experiencias de protesta altamente politizada, en las que la escuela y la universidad se convirtieron en lugares fundamentales de confrontación. (ROSZAK, 1975).

Sin embargo, si queremos analizar las raíces más oscuras del fenómeno, debemos recurrir a la historia italiana de los años del llamado "milagro económico", expresión que caracteriza la rápida industrialización de vastas regiones de Italia después de la reconstrucción posterior a la Segunda Guerra Mundial y la migración interna de los trabajadores que estaban ocupados llenando las industrias del norte en los primeros años '60. El bienestar trajo consigo transformaciones sociales, nuevos estilos de vida, cambios antropológicos como el consumismo y la erradicación cultural de las raíces auténticas del legado tradicional. (TRANFAGLIA, 1989).

Este agitado cambio no correspondía a la necesaria adaptación de los códigos éticos de la sociedad: había un fuerte autoritarismo en la escuela y en la fábrica, junto con la gestión patriarcal de la familia, en la que nunca se cuestionaba el papel del padre.

Este marco, en el que se mezclaba la defensa de la tradición - vista como un baluarte contra la modernización - y la obediencia acrítica a las autoridades, no podía ser aceptado pasivamente por los jóvenes; así, a través de los más altos niveles de escolarización, tomaron posesión de herramientas críticas para combatir los pilares obsoletos de la sociedad en la que vivían. Dos casos en el campo: la lucha política (no separada de la violencia física) o el escape (a menudo hacia paraísos artificiales o utopías anarquistas). Los actores en el escenario de la historia eran, por supuesto, los estudiantes, los que, además de una visión radicalmente diferente, tenían la capacidad de conectarse entre sí y, por lo tanto, dar mayor fuerza a sus reivindicaciones.

La dinámica del choque ya se había hecho evidente en las primeras tentativas de ocupaciones de centros universitarios en los anos 1964-1966, pero asumió un carácter totalmente diferente y se aceleró en el '68: el movimiento estudiantil se convirtió en masa, se extendió en cada universidad y participó en un conflicto radical contra las instituciones académicas y el Estado. La lucha se convirtió en "revolución", con la consecuente destrucción de los espacios para el diálogo y las tentativas de reforma, dejando en el campo sólo la rebelión contra el poder, en las universidades, en la familia y la fábrica. 
En Italia, el acontecimiento histórico del '68 ha desarrollado un papel fundamental tanto en la construcción del icono específico del estudiante rebelde, como de la demonización de los jóvenes, vistos desde entonces con sospecha, al menos por una parte de la sociedad italiana, todavía conformista y con una moral muy rígida, o bien observados con interés por los hidden persuaders (PACKARD, 1957), debido al consumo (por ejemplo, a través de la difusión de ritos y mitos que llegan de Estados Unidos) y a la difusión de la cultura pop, marcada por una fuerte hegemonía anglosajona (las tendencias, la música, la ropa, las drogas). (BONGIOVANNI, 1988).

Más que un análisis sobre la problemática política e ideológica, el interés de la investigación se centra en la relación entre los estudiantes, entendidos como un nuevo sujeto social que aparece de repente sobre la escena italiana, y lo que se puede definir como el storytelling puesto en marcha por los medios de comunicación de masas del momento, el papel impreso, todavía ampliamente influenciado por una moral pequeñoburguesa, que no se esfuerza por entender los motivos reales de las protestas y que se pone contra los jóvenes, adoptando un lenguaje cada vez más crudo y violento para intentar desacreditarlos.

Otra clave para la lectura de los fenómenos que suceden en la historiografía del '68 es la relación entre los jóvenes y los lugares de enseñanza, en este caso las universidades, que son los espacios favoritos para la protesta: destaca una manera distinta de vivir estos no-lugares, de hacerlos suyos, de hacer de los mismos un tipo de ágora para discusiones sobre temas que jamás se habían debatido antes en las aulas universitárias. (AGOSTI; PASSERINI; TRANFAGLIA, 1991).

El análisis se realiza sobre los contenidos (la narración de los hechos) y los mecanismos implícitos en la estrategia de comunicación que, como veremos, tiene como objetivo anular la legitimidad de cualquier protesta de los estudiantes, transformándolos verbalmente en elementos sumamente peligrosos para la sociedad italiana.

La investigación que aquí se presenta se ha llevado a cabo sobre el Corriere della Sera, elegido como diario ajeno a posturas ideológicas o afiliaciones partidistas, en los números que van desde el 1 de enero de 1968 hasta el 30 de junio de 1968, y desvela, a través de un minucioso análisis de los contenidos de los artículos, algunas fases bien identificadas en la manera de contar lo que estaba sucediendo en las universidades y en el movimiento estudiantil.

\section{El '68: las interpretaciones y el debate historiográfico italiano}

Siguiendo el rastro de las múltiples hipótesis interpretativas, antes de entrar de lleno en el meticuloso examen analítico de los acontecimientos y de las opiniones súperestructurales, debemos elaborar una panorámica suficientemente fiable de los muchos y a veces contradictorios significados atribuidos a la reconstrucción de los acontecimientos, bajo el perfil histórico y sociológico. (MARINO, 2004).

La cristalización del '68 se distingue por un énfasis que se eleva a una ritualidad bien codificada: en el espacio cronológico que tomamos en consideración - es decir desde enero hasta junio de 1968 - los medios de comunicación tienden a subrayar, con una repetición obsesiva, algunos elementos que se convierten en "ritos fundados" y que paroxísticamente se vuelven a retomar y a repetir, realizando una precisa selección entre 
hechos y comportamientos que se consideran importantes y esenciales, y otros que, por el contrario, caen, poco a poco, en el olvido por ser considerados poco congruentes con la narración que se pretende presentar a la opinión pública.

La interpretación más fiable, especialmente entre aquellos que vivieron y fueron protagonistas de los hechos, se enfoca, con mucha frecuencia, sobre la construcción intencional del "mito", una estructura simbólica que puede aclarar los motivos implícitos, motivos elevados a explicaciones compartidas y homogéneas dentro de grupos que operaban en la sociedad estudiantil de la época. En este caso, son los actores los que crean una historia sugestiva, y la cuentan, probablemente, no con las características de lo vivido, sino a través del halo fabuloso y degradado de la leyenda. (CAUTE, 1988).

Como suele suceder, se percibe que prevalece especialmente la voluntad de narrar, al poner en marcha las censuras y las omisiones oportunas y al adoptar un punto de vista a posteriori que justifica, legitima, absuelve y santifica la elección de cada uno, elección que se inserta en un marco de espacio-tiempo que une los hechos concretos al cuadro más general, como los fragmentos dispersos de un mosaico, cuya visión y forma aparecen completos sólo cuando los poseemos y ponemos todos en orden.

Un claro ejemplo en este sentido son las corrientes especialmente cargadas de ideología y politizadas de la inteligencia mediática que han interpretado, con la ayuda de gran cantidad de ensayos, artículos y publicaciones, los acontecimientos del '68 italiano, tras su caída, han colocado una etiqueta a tales fenómenos, no como endógenos y autogeneradores del malestar de la juventud en lucha contra la hipocresía de la sociedad italiana, que la relegaba al margen, sino como categoría global de "revolución planetaria", casi conspiratoria, que elimina la peculiaridad italiana. Tal estrategia interpretativa hoy parece claramente inviable, obsoleta y fuertemente criticable puesto que introduce acciones y hechos en una cadena que daba cuentas de una escalada y de una extensa progresión sobre el binomio causa-efecto que en la realidad no era visible y ni siquiera se podía legitimar. Adicionalmente, para apoyar dicha explicación, se hacen totalmente invisibles las variantes locales del movimiento estudiantil y las peculiaridades nacionales, que se muestran absolutamente evidentes, si las miramos a través de una lente de aumento que nos permite observar todos los detalles. Por último, si el tercermundismo y el pacifismo se convierten en categorías, no se comprenden los motivos de las diferentes cronologías del movimiento, las causas deflagradoras para cada uno de los episodios, el denominado "contagio" entre las universidades que, sin embargo, no explica las diversas acciones emprendidas por los estudiantes, y ni siquiera la división - no homogénea en el tiempo de las ocupaciones y de la violencia.

Como ya había identificado correctamente Ortoleva (1988), uno de los conocedores más brillantes y expertos del '68, se necesitan nuevas líneas de investigación a través de las cuales analizar las distintas fases, bien a través de metodologías inusuales (por ejemplo, los ritos de la memoria, solicitando los recuerdos de los protagonistas a través de la praxis autobiográfica), bien ampliando el campo de las fuentes (concretamente la prensa diaria). Además, los acontecimientos se deben reconstruir de forma minuciosa para convertirse plenamente en hechos concretos sobre los que después se pueda realizar la interpretación. No parece que estas sugerencias carezcan de fundamento, puesto que en la mayor parte de ellas hemos presenciado la praxis contraria, es decir, la interpretación ideológica ha prevalecido hasta hoy sobre la descripción puntual y precisa de aquello que ha sucedido 
realmente.

¿Con qué estructuras mentales tenemos que enfrentarnos cuando analizamos la extensa producción ensayística y narrativa sobre el '68? En primer lugar, nos atreveríamos a afirmar que existe una interpretación "en caliente", es decir, la cantidad de ensayos escritos en paralelo al acontecimiento, que, sin embargo, no ofrecen suficientes garantías de imparcialidad y objetividad. En segundo lugar, una visión prospectiva puesta en marcha por aquellos que, escribiendo después de años en relación a su protagonismo en el movimiento de los estudiantes, ha intentado diseñar una visión romántico-idealista de las cosas, afirmando implícitamente que los resultados sociales y políticos de los tiempos más próximos habría que imputarlos - para bien y para mal - a la revolución del '68, que ha derrumbado, mediante su avance, viejos estereotipos y demolido prejuicios atávicos. (BALESTRINI; MORONI, 1988; BILLI, 2001). Por último, pero no menos relevantes, están las narraciones que favorecen puntos de vista totalmente personales, escritas por líderes que, ya en la madurez, deciden contar su parte de la historia y el rol desempeñado en los acontecimentos. (CAPANNA, 1988, 1998, 2008).

En Italia, la densa y compacta producción historiográfica, centrada desde sus inicios en la reconstrucción del annus mirabilis 1968, parece estar más que anulada por una extensa voluntad mitopoyética, que había utilizado desde siempre una estrategia que oscila entre la exaltación de episodios únicos y específicos antepuestos bien pronto a los honores de la crónica - revisados a posteriori para intentar construir una cronología de los hechos de manera racional y consecuente - y al nostálgico recuerdo de aquellos que participaron en la revuelta, quienes, a través del filtro que obnubila la memoria, han contado su existencia, tendiendo a veces a la auto-exaltación de sí mismos como líderes.

En ocasiones recurrentes cada diez años para estudiar y profundizar los acontecimientos de '68, las publicaciones, desarrolladas a través de congresos, jornadas de trabajo y volúmenes misceláneos, han asumido, por tanto, una dimensión cuanto menos heterogénea y polimórfica, en la que podemos encontrar fácilmente algunos hilos conductores que forman parte del animado debate historiográfico. (MARCHETTI, 1988-89). En primer lugar, debemos destacar que todavía está muy presente en algunos historiadores el deseo de elaborar una versión fuertemente influenciada por la ideología en la que se reconocen o con la que se identifican por elección personal o corriente política. Sin duda, los historiadores de izquierda han reconstruido a fondo el paisaje de los años sesenta, dándoles un notable protagonismo, tanto teórico como pragmático, a los que se desplegaron en los grupos comunistas y que desempeñaron un papel importante en la política italiana de los siguientes años setenta y ochenta. Este enfoque abarca la idea de que no había nada espontáneo e individualista en la búsqueda de diversas ocupaciones, sino una voluntad común de quienes, desde el principio, se volvieron receptivos a las palabras clave del "movimiento" y se sintieron unidos por el destino revolucionario. Ellos querían traducir en la práctica el proyecto de la gran revolución contra el autoritarismo, en cualquier forma sería posible conseguirlo.

Una segunda corriente insiste más en una matriz político-cultural derivada de la filosofía de la historia: así, el '68 se legitima y/o se analiza a la luz de una filosofía que favorece una visión iluminada y tercermundista, dirigida a la revolución planetaria. Evidentemente, una estructura conceptual de tal envergadura implica que a un solo acontecimiento no se le atribuye ninguna relevancia excepto para incluirlo en el continuum 
espacio-temporal de la revuelta, y además para legitimar una manifiesta mediación ideológica en el desarrollo y en la concatenación de los fenómenos. La voluntad de poner un orden en las muchas y dispersas piezas del mosaico del '68 lleva a pintar un cuadro impresionista del que no conseguimos percibir cada detalle, pero que resplandece en su totalidad como parte integrante de un mito para la causa sagrada de la rebelión mundial.

Por último, agotado tanto el análisis político como sociológico, vemos otra investigacion de microhistoria de '68: a través de la busqueda en los archivos para recuperar documentos producidos por diversas entidades y sujetos se ententa ireconstruir la historia de las realidades locales, destacando así como en la periferia lejos de las grandes ciudades que eran más atractivas a la protesta debido a la presencia de las universidades, sin embargo, hubo la rebelión y la agitación a menudo provocada por los estudiantes de secundaria.

\section{El miedo frente a la "juventud quemada" en el imaginario social italiano}

¿Por qué reconstruir, bajo un perfil historiográfico y educativo, el alcance y la dimensión del imaginario social italiano configurado en torno a la oleada de protestas $-y$ de divergência - globales desarrolladas por la juventud durante los años sesenta, concretamente, durante el año 1968? El análisis de los mecanismos comunicativos permite refutar el trabajo desacralizador de los medios de comunicación, que fueron plasmando, con una fuerza y un énfasis cada vez mayores, un concepto negativo del binomio estudiante-joven, al adoptar una exagerada simplificación y al asociar cada episodio costumbrista a las presuntas ganas de libertad y rebelión, un mito anárquico y nihilista contagiado también por cierta cinematografía hollywoodiana, bastante de moda en la época (CAVALLUZZI, 1987), como Salvaje (1953) y Rebelde sin causa (1955), verdadero cult movie y mito planetario que, a partir de los años cincuenta, se fue difundiendo entre las nuevas generaciones, hasta convertirse, poco tiempo después, en un fenómeno de masas y código compartido de una fenomenología libertaria que, en parte, se identificaba con los grupos de teddy boys, hijos de las flores, provos.

La simplificación con la que se ofrecía a la opinión pública la figura del joven, despeinado y sin ideales, afincado en el carpe diem y destinado a un fracaso seguro y a la marginación social, se convirtió en el leitmotiv más fiable de la prensa, que no perdió las ocasiones para asociar episodios de micro-criminalidad (consumo de drogas, libertad sexual, fugas de casa, encuentros hippy) a unas protestas bien sólidas, que tenían como finalidad cambiar radicalmente la sociedad italiana, conformista y anclada en viejos esquemas y valores.

Una de las novedades más significativas de los años sesenta (GITLIN, 1987) consistió en un protagonismo nuevo y consciente de los jóvenes universitarios italianos que se hacía visible en "otros" lugares distintos a las tradicionales plazas de la protesta, generalmente representadas por lugares de trabajo y por la mitología de la fábrica con sus ritmos inhumanos. La crítica a la sociedad neocapitalista, alienante y carente de cualquier referencia ética, el descubrimiento de extensas plagas de nuevas pobrezas morales y materiales, pese al consumismo imperante, se encontró desde el principio fluyendo de manera subterránea y cárstica por espacios inusuales y distintos respecto a la lógica habitual del debate político y partidista. Por tanto, se trataba de lugares (aulas, pasillos, patios, carreteras de acceso) que se disponían a convertirse en espacios para derrumbar 
modelos educativos obsoletos y se transformaban en ágora abierta para la discusión y, a veces, también para la violencia, modificando el uso habitual asignado para la actividad académica.

En la amplia iconografía de '68 proporcionada por la prensa diaria, los espacios públicos - en sentido amplio -, particularmente los escenarios universitarios, se convirtieron, por tanto, en otra cosa. Se califican como cartografía de cambio radical, dirigido no sólo a abatir los estereotipos pedagógicos anticuados sino a construir una sociabilidad educativa distinta: las escuelas, las universidad, las asociaciones y los centros culturales se hicieron parte activa de una profunda metamorfosis y modificaron su rol hasta hacerse teatro y caja de resonancia para las protestas contra el blanco favorito de todo el movimiento de '68, es decir, el autoritarismo que impregnaba a toda la sociedad italiana. La participación en los movimientos con convencimiento y entusiasmo, además de la constante presencia en los lugares vividos ahora de manera totalmente distinta a través de las varias y constructivas experiencias de las ocupaciones, expresaba de manera absolutamente radical la complejidad de los procesos sociales que implican de manera total y global a la juventud.

Las nuevas generaciones, nacidas tras la Segunda Guerra Mundial, percibían claramente, mejor que sus padres, natos durante el Fascismo, que el Estado italiano, creado por un impulso ético fortísimo, identificable con la Resistencia, había incumplido las promesas de regeneración moral, cambio global y democratización, que habían sido las palabras claves de la lucha contra el movimiento nazi-fascista. En una sociedad en la que el milagro económico había estado transformando las dinámicas demográficas y las estructuras ocupacionales, el conjunto de la sociedad italiana, con sus jerarquías tradicionales bien consolidadas, sus rituales de poder y sus lógicas políticas gatopardescas, no daba señales de cambio. Desde finales de los años cincuenta, ya eran evidentes (para aquellos que habían observado de cerca el fenómeno) algunos factores que sacaban a la luz esporádicos - y sin embargo claros y manifiestos - comportamientos de revuelta contra los estilos de vida que las élites burguesas difundían como universales y que se podían equiparar. Tal y como ha identificado Simonetta Piccone Stella (1993) en su estudio pionero, tras analizar la cultura que se había creado y divulgado por los denominados hijos del milagro económico, los códigos comunicativos de la rebelión se presentaban con ambivalencia total: por un lado, estos grupos (que se autodefinían como "bandas"), bien identificables por la ropa, las posturas, el peinado y la predominante vida nocturna, aparecían como rebeldes contra los valores negativos de la sociedad de sus padres, y, por otro lado, se convertían en el blanco de la prensa conservadora y de la propaganda capitalista, que los limitaba a una simple categoría mercadotécnica. Los productos y los objetos a través de los que la revuelta creaba sus símbolos, cargándolos de valencias revolucionarias, se convertían, al mismo tiempo, en simples mercancías: los vaqueros (James Dean en Rebelde sin causa), la cazadora de piel (Marlon Brando en Salvaje), las motos (Peter Fonda en Easy Rider: Buscando mi destino), la música pop (el rock and roll de Elvis Presley).

Sorprende la ausencia total, en la prensa diaria, de una voluntad para investigar las instancias más profundas y recónditas de tal rebelión "sin causa" que, en el caso italiano, había que vincularla al contraste entre la impresionante rapidez con la que la modernización, superficial y aparente, del país había modificado la psicología colectiva.

Así aparecieron los jóvenes a los ojos de aquellos que tenían el poder. En realidad, 
la rebelión juvenil estaba dando un salto cualitativo que marcó la trasformación de los fenómenos colectivos de agregación al movimiento de protesta: el centro de este giro es la identificación de un "enemigo" unido a la convicción de que la transformación sólo puede surgir de una fuerte acción colectiva. Hacia mediados de los años sesenta, en el mundo juvenil se produce un cambio, pasando de un anticonformismo genérico a la forma de controversia. La juventud surgió del estado de "rebelión" - simplemente expresando la crisis de las autoridades tradicionales - para convertirse en un "sujeto social" capaz de identificar los propósitos de su lucha. Cuanto más se negaba a incluir a los jóvenes en el interior de la sociedad, más buscaban formas originales para definirse, vestirse y reconocerse; desde ese momento ya no compartirían para nada las categorías éticas y la pertenencia social de sus padres.

Por consiguiente, la brecha generacional ponía en crisis un sistema en el que los lazos se cortaban y se mataba simbólicamente a los padres, considerados responsables del fracaso total de la sociedad. Así, los jóvenes universitarios más progresistas se posicionaban sobre dos frentes contrapuestos e irreconciliables: un "nosotros", positivo, libertario, pacifista, y un "vosotros", negativo, hipócrita, conformista, que no podían encontrar espacios y palabras para compartir, sino solo una irremediable fractura y una dura oposición frontal. (LEONETTI, 1997).

La crítica puesta en marcha por los medios de comunicación pasó a través de la denigración y el desprecio ligado a comportamientos físicos: véase, a título meramente ejemplificativo, por el eco que tuvo sobre las páginas de los periódicos, la etiqueta de "melenudo". (DE MARTINO; GRISPIGNI 1998). Las palabras, con la huella de una lexicografía del todo negativa, están ampliamente apoyadas por un raudal de fotografías que acreditan la imagen de jóvenes al margen, drop-outs, sin objetivos para el futuro, peligrosos para el orden social. (ULIANO, 2008; PASSERINI, 1988-89).

\section{Las protestas estudiantiles universitarias del '68 en la esfera pública italiana}

Las posiciones encontradas en la información se articulan sobre tres líneas prioritarias e interrelacionadas: el debate sobre la ley de reforma universitaria, la protesta de los estudiantes y la crítica, sin apelativo, ejercida contra la revuelta estudiantil.

A partir de diciembre de 1967, es posible encontrar en las columnas del Corriere, con bastante frecuencia, una constante actualización sobre el tortuoso iter legislativo del Decreto-Ley Gui, el denominado "dos pi griego" (identificado con el número 2314 en el debate parlamentario), con la esperanza de que su aprobación podría cambiar el rumbo de las protestas universitarias y enajenar una parte de los motivos que las habían provocado, como la falta de espacios y de aulas, la relación entre el número de docentes y de estudiantes, los criterios de selección para superar los exámenes finales.

La rápida aprobación del Decreto-Ley se presentó como la panacea, la solución para todos los males que acechaban al mundo universitario. Pero era una esperanza frágil, ligada inseparablemente a los acuerdos entre partidos y a la suspensión del debate, por la posibilidad de disolución de las Cámaras en las elecciones de primavera de 1968. Los titulares que aparecieron, casi a diario, desde los inicios de diciembre de 1967, informan de la marcha de las discusiones en las aulas parlamentarias, las posibilidades de mediación entre las exigencias opuestas de los partidos, las críticas planteadas por varios sectores y los Ilamamientos urgentes del ministro Gui. Las dificultades que obstaculizaban su rápida 
aprobación eran muchas y las ponían de manifiesto los exponentes de la mayoría: Rosati (que ocupaba el cargo de responsable del sector escolar de la Dirección Central) afirmaba que "era necesario un compromiso solidario de la mayoría también para exigir un ritmo distinto de trabajo parlamentario, para las sesiones dedicadas a la ley de reforma universitaria [...] y, en caso contrario, a establecer claramente su responsabilidad ante el país". (MEL, 1968). El motivo principal no había que buscarlo - o ya no había que buscarlo más - en la voluntad de modernizar y hacer más eficiente el sector universitario, sino en la urgencia de acabar con las "ocupaciones sistemáticas de las sedes por parte de los estudiantes" que causaban un grave daño a la estabilidad social.

Las palabras del parlamentario Rosati llevan al segundo punto, un topos relevante y ampliamente tratado en las columnas del Corriere: en perfecta sincronía con el farragoso debate del "dos pi griego", se iba montando rápidamente la protesta estudiantil en los recintos universitarios y en las calles y plazas. La aceleración de los acontecimientos resultaba evidente, y hacía temer desórdenes aún más catastróficos.

El eje de las primeras y de las tímidas anotaciones, registradas con cierta perplejidad y sorpresa por el Corriere, fue la revuelta estudiantil en la Universidad Católica de Milán. Sin embargo, hay que afirmar, en honor a la verdad, que las noticias se lanzaron de manera aséptica, mediante una crónica fría, sin dispersiones que prejuzgaran y sin toma de posición; se pensaba, muy probablemente, que se trataba de un fenómeno pasajero de poca importancia. Pero, mientras tanto, se subrayaba que las medidas tomadas por el Rector se habían inspirado en la voluntad de proteger a la "mayoría" de los estudiantes, quienes querían comprometerse y sacar beneficio de la regularidad de las clases, clases que, sin embargo, la reacción de los denominados "150" había interrumpido.

Esta es la historia de los hechos. La universidad había sido ocupada por unos 1200 estudiantes que habían sido expulsados de la policía por la madrugada. Sus reivindicaciones (todas rechazadas por el Senado Académico) se dirigieron a la eliminación del aplastante aumento de impuestos decidido en el verano de 1967, lo que habría penalizado a la mayoría de los estudiantes y la mayor participación de profesores asistentes y académicos en los órganos de decisión. Frente a la imposibilidad de diálogo y al claro rechazo de sus solicitudes, los estudiantes habían decidido cerrar dentro de las instalaciones universitarias y evitar el acceso a los profesores y al rector. Después de horas de gran tensión, la policía logró entrar y dejó a los estudiantes con violencia, quienes entonces habían organizado una procesión para protestar en las calles del centro de Milán.

Las consecuencias de tales gestos no se hicieron esperar: el Senado Académico, a propuesta del rector Ezio Franceschini, había adoptado un castigo punitivo: con un procedimiento de urgencia adoptado por la Autoridad académica, los estudiantes fueron expulsados de la Universidad Católica. (PERO, 2013).

Entonces, mientras la reforma naufragaba entre las dificultades de la hostilidad interpartidista y la responsabilidad de este fracaso ya entonces se asignaba al público ludibrio en las torvas figuras del doble juego de los dirigentes del entonces Partido Comunista, acusadas de querer sacar provecho de los enfrentamientos sostenidos en la plaza - como con claras letras defendía el mismo Gui en un encuentro celebrado en Verona -, las universidades explotaban y la filosofía salía a la escena.

En su muy agudo ensayo On Violence, tras analizar los acontecimientos que habían tenido como protagonistas a los estudiantes de Berkeley, Hannah Arendt sostenía que 
aquella generación parecía caracterizada "en todas partes [...] por su puro coraje, por una sorprendente voluntad de acción y por una no menos sorprendente confianza en la posibilidad de cambios". (ARENDT, 1996, p. 17). Impulsados por motivaciones ideales, ensalzando mitologías a veces equívocas, nutridos por un mix filosófico en el que Nietzsche y Marx se mezclaban sin ningún problema, respetuosos con las banalizaciones que llegaban de los nuevos gurús (Marcuse a la cabeza), los estudiantes pretendían convertirse en el sujeto de la historia y, aunque excluidos de un protagonismo pragmático, se lanzaron a la utopía.

¿Cuánto de esta capacidad de elaborar proyectos se percibe en los periódicos? A juzgar por las páginas del Corriere, diríamos bastante poco. Se podría afirmar que prevalece la voluntad de denigrar y deslegitimar. No por casualidad se suceden, en las columnas que fomentan el alarmismo sobre la protesta, repeticiones paroxísticas y con ánimo de exacerbar sobre las desviaciones del mundo juvenil, sobre los denominados "melenudos" y sobre el uso de las nuevas drogas, una moda que, según se decía, llega de Gran Bretaña, patria conocida en la época por todas las transgresiones, desde el rock hasta las minifaldas, para llegar a los paraísos artificiales generados por los ácidos.

Muy pronto, la opinión pública italiana encontró otros blancos para fomentar la polémica y deslegitimar la actividad de los estudiantes universitarios y, más ampliamente, el carácter inconformista de un número creciente de los jóvenes: así, no sólo los estudiantes, sino su mentor oficial, Herbert Marcuse, fueron objeto de la crítica. Una muestra significativa de esto es un un artículo de Carlo Lorenzi, titulado "Sobre la cresta de la ola", se ironiza sobre aquellos que "cacarean" y "hablan de estructuralismo", citando a Marcuse, definido como el "verdadero testimonio del siglo". Haciendo puntuales referencias al prefacio de la nueva edición americana de Eros and Civilization (MARCUSE, 1966), Lorenzi enfatiza la ambigüedad sustancial del pensamiento del intelectual alemán, quien, tras haber expresado inicialmente un fiel optimismo sobre el futuro del ser humano, se decía, sin embargo, convencido de que la sociedad opulenta había generado "una amplia zona infernal que va desde Vietnam hasta los guetos negros de Nueva York" (LORENZI, 1968), y que ahora ya la confianza se volvía a poner en los países en "vías de desarrollo". En el artículo se critica mucho a Marcuse, tanto por el lenguaje, considerado una moda, como por el cambio de chaqueta operado respecto a los precedentes argumentos socioculturales, que lo habían alzado al rol de líder carismático, cuyas citaciones se habían convertido en un grito de batalla para los estudiantes, junto a Bob Dylan y Joan Baez. De corte bastante similar es el artículo de Enrico Altavilla en el que se informa de las fuertes divergencias ideológicas surgidas entre los estudiantes que participaban de la rebelión en la Alemania federal y sus inspiradores y padres espirituales, entre los que se incluyen Theodor Adorno, Rudolf Augstein, Günther Grass y Jürgen Habermas.

Es fácil poder sacar las debidas conclusiones: se había llegado a un irreparable divorcio entre la filosofía y la protesta. De ahora en adelante, ningún partidario de revueltas podría ya abanderar motivos idealistas, sino solamente un fantasmal deseo de revolución nihilista contra la sociedad burguesa, una especie de cupio dissolvi pesimista, que sacaba partido de una genérica apelación al pensamiento maoísta, o bien de la emblemática figura del Che, o todo el conjunto oportunamente mezclado con la vehemencia verbal de Franz Fanon.

La radicalización del enfrentamiento entre los poderes dominantes dentro de las 
universidades y la voluntad de cambio de los estudiantes encontró su máxima conflagración en los archiconocidos episodios que condujeron a la "batalla de Valle Giulia", considerada el momento álgido del movimiento en Italia (Fig. 01). Tras repetidas apelaciones al diálogo para presentar al rector D'Avack las propias quejas y reivindicaciones, después de haber recibido negativas y rechazos, aproximadamente unos 4000 estudiantes, que salieron de la céntrica Plaza de España, llegaron, la mañana del 1 de marzo de 1968, a la Facultad de Arquitectura de la Universidad de Roma, con el fin de encontrar un espacio para gestionar su asamblea. Una vez en la escalinata que mira hacia la mencionada facultad, se encontraron de frente a un numeroso tropel de fuerzas de policía, alineadas en actitud de guerra, con el fin de impedir la ocupación de los edificios. Los enfrentamientos duraron más de dos horas y media y la violencia ejercida por la policía fue de una gravedad inusual y nunca vista hasta aquel momento. Los estudiantes fueron arrestados, obligados a estar inmóviles, apaleados y llevados a comisaría, donde se les encerró en las salas de seguridad, hasta bien entrada la noche.

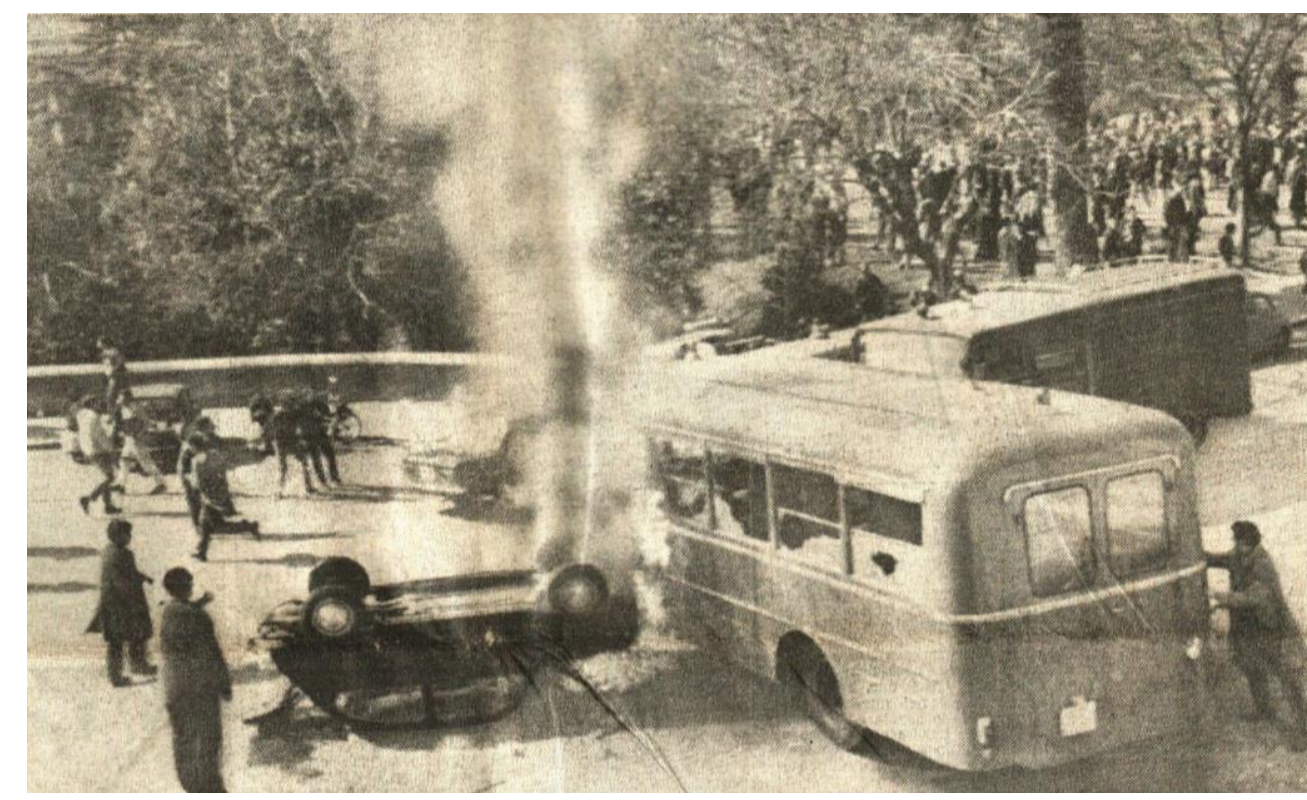

Figura 1 - La "batalla de Valle Giulia", Corriere della Sera, año 93, n. 53, 2 marzo 1968, p. 1.

Fuente: Corriere della Sera, año 93, n. 53, 2 marzo 1968.

Las noticias sobre los enfrentamientos surgidos el 1 de marzo en Valle Giulia fueron ofrecidas por el Corriere con un fuerte alarmismo. El episodio, con tan fuerte despliegue sin una verdadera necesidad, tuvo inmediatamente un eco relevante en los artículos del mencionado diario, cuya redacción no perdió la ocasión para pintar - una vez más - un cuadro de alarmismo y de terror, acompañando, además, las palabras con fotografías de notable impacto emotivo. Testimonio de esta sensación es el espacio atribuido a la crónica de los acontecimientos, siempre colocados en primera página, narrados con abundancia de detalles y acompañados de fotografías.

Como ejemplo de esa estrategia comunicativa, podemos analizar el número publicado el 2 de marzo, tras los hechos de Valle Giulia. En primera página, el artículo de Ascheri tiene como objetivo instilar una fuerte preocupación en la opinión pública, utilizando palabras como "situación dramática", "desórdenes nuevos y graves", dando las cifras de los 
heridos y de los contusionados (47 estudiantes, 150 policías). (ASCHERI, 1968). En las páginas siguientes, se ofrecen otras noticias sobre intervenciones duras de la policía en Turín y en Milán, probablemente para hacer entender que el Estado no está inerme y está reaccionando con mano dura (Fig. 02).

\section{CONTINUA LA TENSIONE ALL'UNIVERSITÀ DI ROMA}

II senato accademico solidale col rettore - Alcuni professori hanno occupato la facoltà di lettere - Altri hanno invece confermato la fiducia a D'Avack - Continua I'azione delle minoranze estremiste presso gli studenti - Migliorano i feriti degli scontri di venerdi

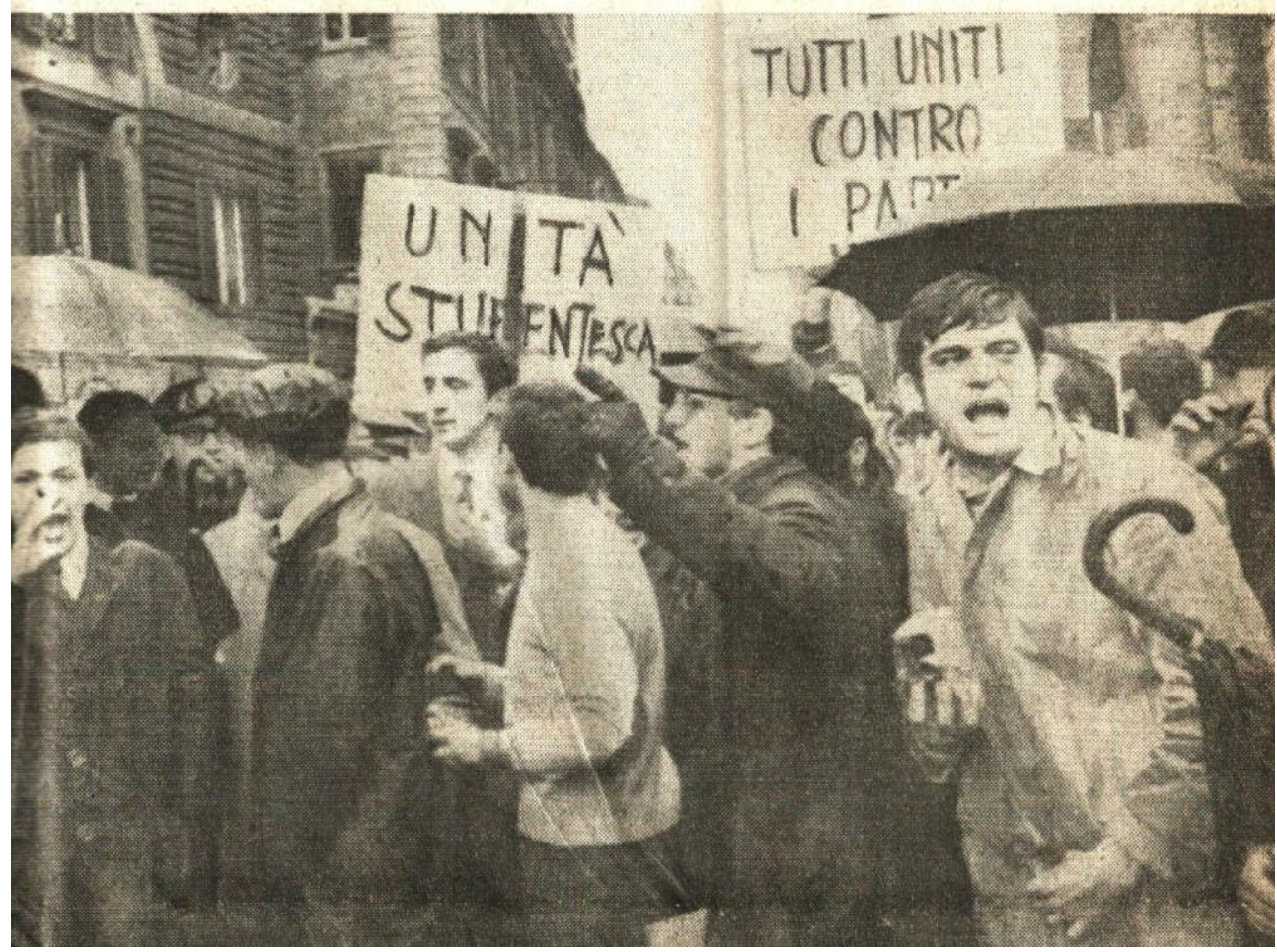

Figura 02 - Corriere della Sera, año 93, n. 54, 3 marzo 1968, p. 1.

Fuente: Corriere della Sera, año 93, n. 54, 3 marzo 1968.

Sin argumento para desmentir, los titulares remitieron al área semántica de la guerra y fueron especialmente indicativos de un sentir común, enfatizando la idea de enfrentamiento de la época y de una "epidemia" que se extendía rápidamente por los demás ateneos italianos. Incluso desde el punto de vista estadístico, las noticias sobre las ocupaciones tuvieron una importancia inusual: el debate sobre el "dos pi griego" pasó a un segundo plano y se consolidó la protesta, que fue, a un tiempo, contada y juzgada. Los estudiantes se convirtieron en "chinófilos", "maoístas", "extremistas", se contaron los "heridos", los "contusionados", se habló de "batalla en la universidad", de "universidad en caos", de "vandalismos", de "desalojos" violentos, de enfrentamientos con la policía, de lacrimógenos y de "detenidos". La geografía de las protestas estudiantiles proporcionó la medida de la amplitud: Roma, Milán, Pisa, Padua, Turín, Perugia, Trieste, Palermo, Catania, 
Venecia, las facultades están ocupadas. En todos los casos, las palabras se cargaron de violencia verbal: en un climax nefasto, las "peleas" se transformaban en "broncas", degeneraban en "violentos enfrentamientos" y en "graves desórdenes". Mientras tanto, el debate de la Ley Gui se encallaba definitivamente en el Parlamento, con recíproco intercambio de acusaciones entre el gobierno y la oposición, considerada la responsable del temporal por meros fines propagandísticos y electorales.

En este estado de caos, la fecha del 12 de marzo de 1968 (y el consiguiente final de la cuarta legislatura y la convocatoria de nuevas elecciones) ratificará el definitivo ocaso del proyecto político de renovar la vida académica y cultural dentro de las universidades italianas.

\section{Conclusiones}

En Italia, los estudiantes universitarios fueron parte de la vanguardia de la lucha por la democratización y modernización de la enseñanza y uno de los sectores de la población más comprometidos con el cambio de los estilos de vida sociales y culturales del país. Reflejaron, asimismo, formas de ser y estar en el mundo y modos de percibirlo y entenderlo en sintonía con lo que acontecía en el panorama internacional, que distaban notablemente de la generación anterior, que incumplió su promesa de ampliar los márgenes de libertad y promover la concordia y la paz internacionales.

Desde el principio, la prensa diaria italiana se hizo eco de las movilizaciones estudiantiles. Pero la imagen ofrecida al público fue, progresivamente, modificándose, al ritmo que la revolución universitaria iba cobrando cuerpo, fuerza y dimensión internacional. Inicialmente, se presentaron las protestas estudiantiles como un movimiento universitario inicuo, como algo anecdótico, pasajero, que se diluiría por sí mismo. Pero, poco a poco, la imagen pública de los jóvenes transformando y haciéndose más dura. Hasta tal punto que los estudiantes se presentaron como una especie de delincuentes, y las protestas como una guerra entre aquéllos y las instituciones y las fuerzas del orden público. De este modo, la prensa italiana, respondiendo a los intereses de la burguesía y las oligarquías, pretendió hacer ver que los universitarios que participaban de las revueltas eran vagos y maleantes, que hacían uso de las drogas y, por tanto, no podían tener razones para reivindicar otra universidad.

Pero, a pesar de todo, la acción de los estudiantes universitarios italianos desarrollada durante finales de 1967 y el primer semestre de 1968 cosecharon notables éxitos en la desarticulación de las estructuras organizativas y de representación vigentes en las facultades y lograron incidir en la trayectoria de la política general de la educación del país. De este modo, Italia se sumó a la corriente internacional de militancia estudiantil y movilizaciones organizadas, acompañadas, en muchos casos, del liderazgo de profesores, que desde mediados de la década de 1960, fueron en progresivo aumento, hasta que, ya durante las dos siguientes décadas, se convirtieron en factores determinantes del triunfo de los procesos de liquidación de algunas dictaduras, como las de Argentina, Brasil, Chile, España, Grecia, Portugal y Uruguay.

\section{Fuentes: Corriere della Sera (1968) [en orden cronológico]}

MARANINI, G. (7 enero). Il progetto di riforma per ora è solo fonte di confusione. 3, p. 2. 
MEL, E. (3 febrero). Forse la riforma universitaria alla prossima legislatura. 29, p. 2 ROBERTAZZI, M. (9 febrero). Le agitazioni negli atenei. 34, p. 14.

MEL, E. (10 febrero). La maggioranza parlamentare per la riforma universitaria. 35, p. 17. ALTAVILLA, E. (11 febrero). Hanno perso i loro profeti i terribili goliardi tedeschi. 36 , p. 3. DE TISI, C. (16 febrero). II malcontento degli universitari. 40, p. 14.

ENRIQUES, G. (21 febrero). I mali dell'università. 44, p. 5.

ASCHERI, G. (25 febrero). Occupate dagli universitari altre due facoltà romane. 48, p. 1.

ASCHERI, G. (1 marzo). Violenti scontri tra studenti e polizia dopo lo sgombero dell'università di Roma. 52, pp. 1-2.

ASCHERI, G. (2 marzo). Intervento della polizia all'università di Torino. 53, p. 2.

ASCHERI, G. (2 marzo). Nuovi gravi disordini a Roma con 200 tra contusi e feriti. 53, pp. 1-2.

ASCHERI, G. (3 marzo). Continua la tensione all'università di Roma. 54, p. 1.

BIANCHI, L. (5 marzo). II governo proporrebbe il nuovo provvedimento per gli atenei. 55, p. 2.

MARCATO, R. (8 marzo). Violenti incidenti a Torino per un corteo di studenti. Scontri a più riprese con gli agenti. Numerosi i feriti e i contusi, tra i quali un docente. 58, p. 1.

\section{Referencias}

AGOSTI, A.; PASSERINI, L.; TRANFAGLIA, N. (Eds.). La cultura e i luoghi del '68. Milano: Angeli, 1991.

ALDGATE, A.; CHAPMAN, J.; MARWICK, A. (Eds.). Windows of the Sixties: exploring key texts of media and culture. London, New York: Taurus, 2000.

ARENDT, H. Sulla violenza. Tr. it. Parma: Guanda, 1996.

BALESTRINI, N.; MORONI, P. L'orda d'oro. 1968-1977. Milano: SugarCo, 1988.

BELLUCCI, S. II movimento studentesco del Sessantotto. Cause e condizioni di sviluppo.

Sociologia - La società in ... rete, 2, 3, p. 34-55, 2008.

BILLI, F. (Ed.). Gli anni della rivolta. 1960-1980: prima, durante e dopo il '68. Milano: Punto Rosso, 2001.

BONGIOVANNI, B. Società di massa, mondo giovanile e crisi di valori. La contestazione del '68. In: TRANFAGLIA, N.; FIRPO, M. (Eds.). La storia. I grandi problemi dal Medioevo all'Età Contemporanea, v. VII, L'Età Contemporanea, t. 2, La cultura. Torino: Utet, 1988. p. 671-694.

BRAMBILLA, M. Dieci anni di illusioni. Storia del Sessantotto. Milano: Rizzoli, 1994. BRECCIA, A. Le istituzioni universitarie e il Sessantotto. Bologna: Clueb, 2013. CAGNOLATI, A. "Ma che colpa abbiamo noi?". Mass-media e protesta studentesca. In: BETTI, C.; CAMBI, F. (Eds.). II '68: una rivoluzione culturale tra pedagogia e scuola. Itinerari, modelli, fronteire. Milano: Unicopli, 2011. p. 187-193.

Decostruzione simbolica e mitopoietica: le università italiane durante il ' 68 (Conferencia presentada al Convegno di studi del Centro italiano per la ricerca storico educativa - Cirse, Sguardi della storia. Luoghi, figure, immaginario e teorie dell'educazione, Bologna, 26-27 febbraio 2016), 2016. 
CALABRESE, O. Appunti per una storia dei giovani in Italia. In: ARIÈS, P.; DUBY, G. (Eds.). La vita privata. II Novecento. Roma-Bari: Laterza, 1988. p. 79-106.

CAPANNA, M. Formidabili quegli anni. Milano: Rizzoli, 1988.

II Sessantotto al futuro. Milano: Garzanti, 2008.

Lettera a mio figlio sul sessantotto. Milano: Rizzoli, 1998.

CATALANO, F. I movimenti studenteschi e la scuola in Italia (1938-1968). Milano: II Saggiatore, 1969.

CAUTE, D. Sixty-Eight. The Year of the Barricades. London: Hamish Hamilton, 1988.

CAVALLI, A.; LECCARDI, C. Le culture giovanili. In: Storia dell'Italia repubblicana. v. III, L'Italia nella crisi mondiale. L'ultimo ventennio, t. II, Istituzioni, politiche, culture. Torino:

Einaudi, 1997. p. 709-800.

CAVALLUZZI, R. II potere delle immagini. Cinema e sessantotto. Bari: Dedalo, 1987.

CHIAPPANO, A.; MINAZZI, F. (Eds.). Anno Domini 1968. L'immaginazione che voleva il potere. San Cesario di Lecce: Manni, 2004.

CHIARANTE, G. La rivolta degli studenti. Roma: Editori riuniti, 1968.

COCCIA, B. (Ed.). 40 anni dopo: il Sessantotto in Italia fra storia, società e cultura. Roma: APES, 2008.

D'ERAMO, M. (Ed.). L'immaginazione senza potere: mito e realtà del '68. Roma: Mondo operaio-Avanti, 1978.

DE MARTINO, G.; GRISPIGNI, M. I Capelloni. Mondo Beat, 1966-1967. Storia, immagini, documenti. Roma: Castelvecchi, 1997.

FERRY. L.; RENAUT, A. La pensee 68: essai sur l'anti-humanisme contemporain. Paris: Gallimard, 1985.

FEUER, L. S. The Conflict of Generations: The Character and Significance of Students Movements. New York, London: Basic Books, 1969.

FLORES, M.; DE BERNARDI, A. II Sessantotto. Bologna: II Mulino, 1998.

FRASER, R. (Ed.). 1968: A Student Generation in Revolt. New York: Pantheon, 1988.

GIORGI, A.; MINEO, L. Dal "gran vento di maggio" a "una miriade di gruppetti". Fonti documentarie per lo studio dei movimenti di contestazione (1966-1970). Ventunesimo secolo, XIII, 34, p. 19-48, 2014.

GITLIN, T. The Sixties: Years of Hope, Days of Rage. New York: Bantam, 1987.

GUARNACCIA, M. Beat e mondo Beat: chi sono i Beats, i Provos, i Capelloni. Viterbo: Stampa Alternativa, 2005.

HERNÁNDEZ HUERTA, J. L.; GONZÁLEZ GÓMEZ, S. Opinión pública y educación durante la transición a la democracia en Argentina. Primeras consideraciones y guía de fuentes documentales. Educació i història: Revista d'història de l'educació, 24, p. 173215, 2014.

HERNÁNDEZ HUERTA, J. L.; ORTEGA GAITE, S. Civic Education and public opinion in Argentina during the transition to democracy (1982-1983). History of Education \& Children's Literature, 10 (2), p. 359-389, 2015.

HILWIG, S. J. 'Are you calling me a fascist?' A Contribution to the Oral History of the 1968 Italian Student Rebellion. Journal of Contemporary History, 36 (4), p. 581-597, 2001. 
KURLANSKY, M. '68. L'anno che ha fatto saltare il mondo. Tr. it. Milano: Mondadori, 2004.

LEONETTI, F. (Ed.). Le scelte del Sessantotto. Milano: Associazione culturale Leoncavallo libri, 1997.

LEVITT, C. Children of Privilege: Student Revolt in the Sixties. A Study of Student Movements in Canada, the United States, and West Germany. Toronto: Toronto UP, 1984. MARCHETTI, A. Le interpretazioni del '68. In: Il Sessantotto: I'evento e la storia, Annali della Fondazione Micheletti, 4 (1988-89), p. 11-19.

MARCUSE, H. Eros e civiltà. Tr. it. Torino: Einaudi, 1996.

MARINO, G. C. Biografia del Sessantotto: utopie, conquiste, sbandamenti. Milano: Bompiani, 2004.

MARWICK, A. The Sixties: Cultural Revolution in Britain, France, Italy, and the United States, ca. 1958-1974. Oxford, New York: Oxford UP, 1998.

MASSARI, R. II '68. Come e perché. Bolsena: Massari editore, 1998.

MORDENTI, A. (Ed.). Come eravamo: documenti fotografici per una storia delle lotte studentesche a Roma: 1966-1972. Roma: Savelli, 1975.

MORDENTI, R. Frammenti di un discorso politico. II '68, il '77, l'89. Verona: Essedue edizioni, 1989.

MOVIMENTO STUDENTESCO. Libro bianco dell'Università Cattolica di Milano. Milano: Ed. Relazioni social, 1968.

ORTOLEVA, P. I movimenti del '68 in Europa e in America. Roma: Editori Riuniti, 1988. PACKARD, V. The Hidden Persuaders. New York: McKay, 1957.

PASSERINI, L. II '68 nei processi di comunicazione intersoggettiva. In: II Sessantotto:

l'evento e la storia, Annali della Fondazione Micheletti, 4 (1988-1989), p. 1-9.

PERO, L. II movimento degli studenti nell'Università Cattolica di Milano dal 1967 al 1969. In: BRECCIA, cit., p; 81-94, 2013.

PICCONE STELLA, S. La prima generazione. Ragazze e ragazzi nel miracolo economico italiano. Milano: Angeli, 1993.

POGGIO, P. P. (Ed.). II Sessantotto: l'evento e la storia. Atti del convegno: 1968-1988: venti primavere dopo, un bilancio, tenutosi a Brescia, 9-11 marzo 1989. Annali della Fondazione Micheletti, 4 (1988-1989).

PORTELLI, A. The Battle of Valle Giulia: oral history and the art of dialogue. Madison: University of Wisconsin Press, 1997.

ROSSANDA, R. L'anno degli studenti. Bari: De Donato, 1968.

ROSTAGNO, M. (Ed.). Università: I'ipotesi rivoluzionaria. Padova: Marsilio, 1968.

ROSZAK, T. La nascita di una controcultura: riflessioni sulla società tecnocratica e sulla opposizione giovanile. Tr. it. Milano: Feltrinelli, 1975.

RUGGIERO, G. Maggio e dopo: motivi e problemi della contestazione giovanile. Milano: Mursia 1970.

TARROW, S. Democracy and disorder: protest and politics in Italy, 1965-1975. Oxford: Clarendon Press, 1989.

TOLOMELLI, M. II Sessantotto. Una breve storia. Roma: Carocci, 2008. 
TRANFAGLIA, N. (Ed.). Crisi sociale e mutamento dei valori. L'Italia negli anni Sessanta e Settanta. Torino: Tirrenia Stampatori, 1989.

ULIANO, L. (Ed.). '68. Un anno di confine. Milano: Rizzoli, 2008.

VAN DIJK, T. A. Análisis del discurso ideológico. Versión, 6, p. 15-43, 1996.

. El análisis crítico del discurso. Anthropos, 186, p. 23-36, 1999.

VIALE, G. II Sessantotto. Tra rivoluzione e restaurazione. Milano: Mazzotta, 1978.

ANTONELLA CAGNOLATI é Doutorada em História da Cultura Europeia e professora de História da Educação no Departamento de Estudos Humanísticos da Universidade de Foggia (Itália). É membro de comitês científicos de coleções e revistas internacionais. Ela é membro do Conselho de Administração do Centro Italiano per la Ricerca Storico-educativa (Cirse) e vice-presidente da Associação Universitária para o Estudo das Mulheres (Audem). Faz parte das Sociedades científicas, a Conferência Permanente Internacional sobre a História da Educação (Ische), Sociedade Espanhola de História da Educação (Sedhe).

Endereço: Departamento de Estudos Humanísticos - Universidade de Foggia - Via Arpi, 176 - 71121 - Foggia - Itália.

E-mail: antonella.cagnolati@unifg.it

Recebido em 02 de outubro de 2017.

Aceito em 28 de novembro de 2017. 